\title{
Reflections on clinical research in sub-Saharan Africa
}

Irene Kuepfer, Christian Burri

Swiss Tropical and Public Health Institute, Department of Medicine, Socinstrasse 57, CH-4002 Basel, Switzerland

Küpfer, Irene Sylvie, Improved melarsoprol therapy for "Trypanosoma brucei rhodesiense" sleeping sickness. 2009, Doctoral Thesis, University of Basel, Faculty of Science.

Article published in the International Journal for Parasitology

Int J Parasitol. 2009 Jul 15;39(9):947-54. doi: 10.1016/j.ijpara.2009.02.021. Epub 2009 Mar 24.

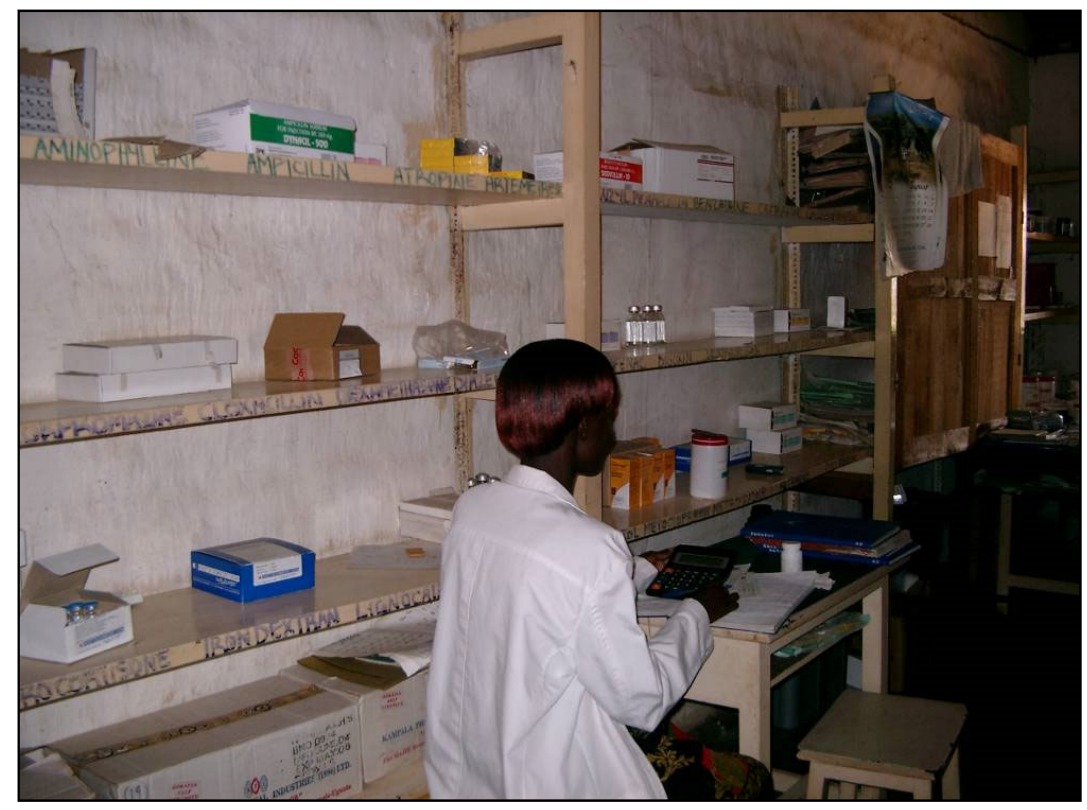

Pharmacy at the Lwala Hospital, Uganda 


\begin{abstract}
The urgent need for new, safe and sustainable interventions against diseases that disproportionally affect the poor is finally receiving global attention and the funding landscape for development projects has significantly improved during the past decade. For the development of new drug and vaccine candidates, clinical trials have become the most important tool to assess their safety and efficacy. Recently, there has been a seismic shift in the number of clinical trials conducted in resource-limited settings. We discuss the current framework of clinical research in sub-Saharan Africa, from building product pipelines to the capacities needed for the conduct of trials according the harmonized Good Clinical Practice (GCP) ICH E6 guideline. We place emphasis on clinical research in neglected tropical diseases which still frequently has to be conducted with limited financial, logistical and human resources. Given those short-comings we recommend minimum standards needed at the local, national and sponsor levels to provide GCPcompliant clinical research.
\end{abstract}

Keywords: Neglected tropical diseases; Sub-Saharan Africa; Research and development; Clinical research; Good clinical practice (GCP); Minimum standards 


\section{Introduction}

Clinical studies and trials are the most important tools to assess the evidence of new medical interventions including drugs and vaccines. The correct and fair conduct of the investigations is essential and this was one of the key messages of the first International Clinical Trials Day of $20^{\text {th }}$ May 2005 which was organized to boost clinical research worldwide (http://www.ecrin.org).

Almost 10 years ago, the term ' $10 / 90$ gap' was coined, recognizing that in the preceding 30 years only $10 \%$ of global health research has been dedicated to diseases that accounted for $90 \%$ of the global disease burden (1). Fortunately, since 2000 the funding landscape for tropical disease research has significantly improved (2) and as a result, the number of clinical trials conducted in sub-Saharan Africa has multiplied. Today, a new challenge in certain fields is coordination of the efforts of numerous global initiatives and consortia contributing to new, sustainable interventions against diseases which disproportionately affect the poor.

During the same period, international rules for the conduct of trials have been advanced from guidelines to laws in the Western world and largely implemented. The harmonized Good Clinical Practice (GCP) ICH E6 guideline (3) set a quality standard, but has also added an unprecedented dimension of complexity to clinical research. Whereas many sub-Saharan drug authorities are still not in the position to impose fully GCP-compliant trials, most international funding agencies and sponsors do so and clinical research in resource-limited countries has to satisfy international laws and regulations. This has the advantage of increasing quality standards and credibility of the data produced, but may also lead to conflicts with cultural, political and socio-economic facts and values.

Trials on interventions against rare diseases or those which have a mainly rural distribution often have to be carried out with limited finances, logistics and human resources. It stands to reason that finding the equilibrium between those different realities remains a challenge. Those conditions and settings also stand in sharp contrast to the growing number of high standard research centers in sub-Saharan Africa. In this article we review the current framework and the practical aspects of clinical research in subSaharan Africa with an emphasis on clinical trials for the most neglected diseases. Given the various challenges and restrictions of such research in collaboration, we attempt also to present minimum standards for the appropriate conduct of clinical trials and studies.

\section{Creating and maintaining a pipeline of new interventions against neglected tropical diseases}

\section{Neglected tropical diseases}

Respiratory infections and diarrheal diseases are the two main categories of infectious diseases responsible for a high burden of disease in resource-poor settings (http://www.who.int/mediacentre/factsheets/fs310_2008.pdf). In addition, the "Big Three" - HIV/AIDS, malaria and tuberculosis account for 5.6 million deaths and the annual loss of 166 million disabilityadjusted life years (DALYs) (4). Premature mortality and high morbidity are also caused by another category of illnesses referred to as neglected tropical diseases, e.g. Human African Trypanosomiasis, Chagas disease, schistosomiasis, leishmaniasis, dengue fever and leprosy. Together those diseases cause approximately 534,000 deaths annually and are responsible for a very large number of years of life lost as a result of from premature disability (DALYs). Some estimates suggest that the neglected tropical diseases result in 57 million DALYs lost annually, a number that is almost as high as that of each of the 'Big Three' (4).

Neglected tropical diseases share three common characteristics. Firstly, they are only prevalent in resource-limited settings and hence the market incentives are far too low to trigger corporate investments for new interventions. Second, the target product profile of new diagnostics and chemotherapies for such diseases must allow their use under very difficult field conditions and be stable under extreme conditions of heat and humidity (5). Third, the interventions need to be cost-effective to have a fair chance to be delivered to the target population by the typically weak health systems or non-governmental organizations (NGOs). 
Undoubtedly, we are significantly lacking the tools to properly diagnose and treat many neglected tropical diseases. The withdrawal of pharmaceutical companies from tropical disease research and development (R\&D) in the 1970s left a very large gap in the development of new and affordable drugs (6). From 1975 to $1999,1,393$ new pharmaceuticals were marketed worldwide of which only 13 were against tropical diseases (7), mainly for malaria and tuberculosis.

\section{The new landscape after the year 2000}

The landscape for neglected disease $R \& D$ has dramatically changed since the beginning of the new millennium (2) and the reasons are manifold. An in-depth analysis performed in 2004 showed that 63 neglected disease drug projects were under way, including two new drugs in registration status and 18 new products in clinical trials, half of which were already in Phase III. With sufficient funding these projects were expected to deliver eight to nine new neglected-disease drugs until 2009, even if no further projects would have commenced after this (2).

\section{Searching for new medical interventions against neglected tropical diseases}

Due to the recognized need for new, rapid and sustainable interventions, and due to lengthy and expensive pharmaceutical development processes, short- and long-term strategies are combined in order to deliver new drugs and vaccines to this neglected market. One of the central pillars is the extensive screening of existing compound libraries. Rapid success and a comparatively low attrition rate can be expected from compounds which have already undergone pre-clinical and/or partial clinical development but were abandoned by industry due to economic reasons or inefficacy for the original indication. Unfortunately, such compounds are hard to come by, possibly due to potential embarrassment should the compound be successful and the fear of important trade secrets being passed on unintentionally. Other shorter-term strategies involve the development of follow-on drugs in the same class, fixed dose combinations of existing drugs, and of new pediatric formulations to make childhood treatment easier. Exciting results can also come from re-directing compounds developed for other diseases towards neglected diseases, also called "therapy-switching" or "piggy-backing" (8).

However, to create a sustainable pipeline of compounds, long-term strategies are important. There the focus is on "breakthrough" innovation; i.e. novel compounds with a novel mechanism of action against the pathogens (2). This approach is of primary importance to cope with the increasing problem of resistances in diseases where treatments exist (e.g. malaria and tuberculosis) but also to find acceptable therapies for those diseases where acceptable solutions are so far lacking (e.g. sleeping sickness, leishmaniasis). A recent example of drug resistance is the report of artemisinin-resistant malaria in western Cambodia (9).

On the positive side, a number of new screening centers tackling the above-mentioned tasks became operational during the past few years and the number of hits and lead compounds is increasing. However, a major challenge and persisting bottleneck appears to be the professional selection of the leads and particularly the advancement of the most promising compounds into funded professional pre-clinical programs.

\footnotetext{
Alternative business models for $R \& D$ in tropical diseases

Private sector investments in R\&D are not triggered by commercial incentives, thus a system that not only shares the risk but also the cost of a highly expensive undertaking was established and became known as Public Private Partnerships (PPPs), now called Product Development Partnerships (PDPs). Examples of PPPs/PDPs such as the special program in research and training of tropical diseases (TDR) date back to 1975. Today, the development of new partnerships has accelerated and examples are numerous. The Initiative on Public Private Partnerships for Health lists over 90 such organizations in its database (http://www.globalforumhealth.org).

These organizations mostly operate like virtual drug companies and are largely responsible for the recent seismic shift in R\&D for tropical diseases (6). At present the range of funding is mainly provided by philanthropic organizations led by the Bill \& Melinda Gates Foundation (http://www.gatesfoundation.org). In 2005, philanthropic organizations contributed over $78 \%$ of the total funding of R\&D partnerships for neglected diseases, whereas public funding was calculated at a mere $16 \%$.
} 
Public funding has a long history at the National Institutes of Health (NIH, USA) mainly in the form of competitive research grants, whereas other institutions such as the Institut Pasteur (France) or the Medical Research Council (MRC, United Kingdom) made targeted investments in satellite centers. Broad public funding is a major factor for sustainable tropical disease $R \& D$, as only governments can guarantee a longterm commitment (10). The report of the World Health Organization's (WHO's) commission on public health, innovation and intellectual property rights released in April 2006, urged the WHO to develop a global plan of action to secure enhanced and sustainable funding for developing and making accessible products to address diseases that disproportionately affect developing countries (11).

An example of targeted public funding is the European \& Developing Countries Clinical Trials Partnership (http://www.edctp.org) which was created in 2003. During the past year it has gained substantial momentum, funding drug and vaccine development in HIV/AIDS, malaria and tuberculosis. Areas where governments can indirectly encourage corporate interest in rare and/or neglected diseases are indemnification strategies. Since 1983 the U.S. Food and Drug Administration (FDA) has offered tax incentives for clinical trials as well as 7 years of marketing exclusivity for drugs developed for rare diseases in the US through the Orphan Drug Act (http://www.fda.gov/orphan/oda.htm). In 2007, the U.S. Congress approved an amendment to the FDA Revitalization Act

(http://www.fda.gov/oc/initiatives/HR3580.pdf), which created a transferable voucher to encourage treatments for tropical diseases. The sponsor of a newly approved drug which prevents or treats an eligible tropical or neglected disease will receive a priority review voucher, which can then be transferred to the submission of another human drug or sold to another company. Priority review reduces the time period for the review of the registration dossier from an average of 18 months to no longer than 6 months. Economists estimate that the priority review voucher used for a potential blockbuster drug could be worth more than US\$ 300 million. In comparison, the average costs of developing a new chemical entity were estimated to be US\$ 800 million in the year 2003 including capitalization. The voucher could thus enable a company to recoup a significant portion of the cost of developing a new drug (http://www.iavi.org/viewfile.cfm?fid=47963). This seemingly straight forward system awaits its first application, with some critical voices cautioning against potential misuse or a decreased quality of the review process (12).

In 2000 the European Union adopted the Orphan Medicinal Products legislation under which companies with an orphan designation for a medicinal product benefit from incentives such as protocol assistance (scientific advice during the product-development phase); marketing authorization (10-year marketing exclusivity); financial incentives (fee reductions or exemptions); and national incentives detailed in an inventory made available by the European Commission

(http://www.emea.europa.eu/pdfs/human/comp/29007207en.pdf). The European Union pharmaceutical legislation may support authorities that lack regulatory capacity; those authorities can obtain scientific opinion from the European Medicines Evaluation Agency (EMEA) through WHO on products intended exclusively for markets outside the community (13).

Last but not least, the pharmaceutical industry has renewed its interest and engagement in tropical diseases. Examples for new industry sponsored research facilities are the Novartis Institute for Tropical Diseases (http://www.novartis.com/research/nitd), GlaxoSmithKline's Diseases of the Developing World Initiative (http://www.gsk.com/research/about/about_diseases.html), Sanofi-Aventis' Malaria Initiative (http://en.sanofi-aventis.com/sustainability/sustainability.asp) and Astra-Zeneca's (http://new.tballiance.org /newscenter/view-brief.php?id=52) effort in tuberculosis research. These institutions will have a major impact in coming years (6).

\section{Persistent and emerging complexities - intellectual property rights}

A key aspect of cooperative R\&D is the role of intellectual property protection mechanisms (IPPM). Through adequate management of the resulting intellectual property (IP), the public sector can benefit from its R\&D investments through the availability of the most modern products with conditions that are beneficial for the developing world, eliminating otherwise significant barriers to access (14). Joint research ventures such as partnerships among developing countries, the private sector, academic institutions and NGO's depend on IP management strategies. However, recent publications indicated that intellectual property rights negotiations are more complex in horizontal research joint ventures (same industry) and when universities are involved (15). 
Patents are the most frequently used IPPM (15). For example, the Medicines for Malaria Venture (MMV) arrangement for the synthetic peroxide antimalarial project involved the assignment of a patent from a U.S. university. The patent included claims for treating cancer and schistosomiasis, which are retained by MMV. The compound was licensed to an Indian company, Ranbaxy, with a provision for reversion of the rights to MMV should Ranbaxy fail to meet certain milestones (such as meeting public sector demand in target developing countries at affordable prices). Likewise, further segmentation of the market into a "traveler's market" and worldwide private sector sales provides potential commercial incentives for Ranbaxy once they meet the criteria for public health interest (14). But innovative partnerships have also come up with non-patented drugs. The Drugs for Neglected Diseases Initiative (DNDi) partnered with French pharmaceutical company Sanofi-Aventis in 2007 and brought fixed-dose artesunate-based combination therapies to the African market. This patent-free model is now also implemented for delivering anti-malarial treatment to South American patients (http://www.ip-

watch.org/weblog/2008/04/17/innovative-partnership-to-create-another-patent-free-malaria-drug).

\section{Capacity building}

During the past two decades, numerous research centers have been developed in sub-Saharan Africa, where high quality research is conducted. The majority has strong links with a Northern partner and the number of regional and supra-regional networks of excellence is growing. In addition, many of the centers are linked to patient cohorts or even demographic surveillance systems (http://www.indepthnetwork.org). However, there exists a substantial regional imbalance with the majority of these centers being located in South and East Africa, a few in West Africa (http://www.africaclinicaltrials.org) and only limited activities exist in Central Africa. Furthermore, most of these centers are involved in research in the area of malaria and HIV/AIDS or tuberculosis, but almost never in neglected diseases.

Many of the research centers have evolved from being trial sites conducting epidemiological studies or single trials to project sites, and some have become research centers with all the capacities needed to maintain a portfolio of trials and to be involved in different fields of activity (see Table 1).

Table 1. Characteristics of the evolution from a trial site to a research centre (16)

\begin{tabular}{|c|c|c|c|}
\hline & Trial site & Project site & Research centre \\
\hline What is core? & $\begin{array}{l}\text { Informal alliance of } \\
\text { projects and trial sites }\end{array}$ & $\begin{array}{l}\text { Established infrastructure } \\
\text { to projects/trials }\end{array}$ & $\begin{array}{l}\text { Fully established entity that } \\
\text { provides all science } \\
\text { infrastructure }\end{array}$ \\
\hline Core funding & $\begin{array}{l}\text { Resources from projects } \\
\text { fund the core }\end{array}$ & $\begin{array}{l}\text { Small amount of core - } \\
\text { funding and resources }\end{array}$ & $\begin{array}{l}\text { Funding of core established } \\
\text { and projects contribute to } \\
\text { core }\end{array}$ \\
\hline Portfolio & $\begin{array}{l}\text { Single or small number } \\
\text { of projects that drive } \\
\text { core }\end{array}$ & $\begin{array}{l}\text { Small number of projects - } \\
\text { able to add different } \\
\text { diseases or interventions }\end{array}$ & $\begin{array}{l}\text { Different interventions } \\
\text { and/or } \\
\text { different diseases }\end{array}$ \\
\hline Time focus & Short term & Mid term (3-5 years) & Long term $(>10$ years $)$ \\
\hline Infrastructure & $\begin{array}{l}\text { Very basic and } \\
\text { dependent } \\
\text { on project funding }\end{array}$ & $\begin{array}{l}\text { Established basics that } \\
\text { survive } \\
\text { individual trials/projects }\end{array}$ & $\begin{array}{l}\text { Full infrastructure } \\
\text { maintained over time with } \\
\text { projects paying share }\end{array}$ \\
\hline
\end{tabular}

The development of a trial site to a research centre is a long process in which well-planned and sustainable capacity building has a central function. A critical mass of local researchers needs to be trained and given a career perspective to avoid excessive "brain drain"; the institution must build up the leadership to not only deliver research excellence, but also provide adequate governance, administrative, financial and management functions (17); the respective infrastructures needs to be built; and the quality assurance systems necessary for the compliant conduct of the trials must be implemented and enforced. In the experience of our team, which is routinely involved in monitoring and auditing of a substantial 
number of trials and research centers, this latter aspect still needs attention. The level of detail requested today is considerable and the main findings include the confusion of document versions, incomplete tracking of processes and unwanted deviations from protocols or procedures. Clear and practical internal standard operating procedures at all levels and the installation of internal quality control help to avoid such verdicts.

The creation of such sustainable and comparatively independent research centers may not always be achievable. Many of the most neglected diseases (e.g. human African Trypanosomiasis, Buruli ulcer, trachoma) are characterized by a rural distribution and may be locally controlled or even eliminated through dedicated disease control activities. Conducting clinical trials in such regions requires the involvement of small treatment facilities with often very basic infrastructure and very limited human resources. The conduct of a single clinical trial often leads to a significant reduction in the relevant patient number in the catchment area, preventing follow-up projects. The switch to other diseases or projects is normally impossible due to the remoteness of the center and the mentioned limitations.

Particular attention has to be paid in such situations to the appropriate information about the intentions of the researchers, the adequate training of local staff and site improvement. The minimum obligation is to leave behind a team well-trained in the relevant areas and a laboratory with specific improvements for the daily routine.

\section{The conduct of clinical trials in sub-Saharan Africa}

\section{Ethics}

The goals of research are always secondary to the well-being of the participants. This requirement is made clear in the Declaration of Helsinki and is regarded as the fundamental guiding principle of research involving human subjects (18). Discussions on bioethics of clinical research in resource-limited settings are manifold and include the therapeutic areas, the quality and quantity of research as well as discussion on the use of placebo or the use of the best versus the locally available standard of care.

Guidelines on bioethics and clinical research are numerous and sometimes conflicting. Where the Declaration of Helsinki has established the guiding principles of research involving human subjects, it does not cover all relevant aspects for the conduct of clinical research. Currently, the correct overall reference for the conduct of clinical trials is being discussed. The guiding role of the Helsinki Declaration was contested by the U.S. FDA and it was decided to replace it by the standards described in the ICH GCP (19). On the other hand the ICH guideline itself had been seen as being too comprehensive and strict for the conduct of clinical trials in resource-limited countries (20). The increase in clinical trials conducted in such settings goes hand in hand with an increasing capacity and this discussion will continue with a yet unknown outcome.

In this article, we only discuss ethical aspects that have a direct and/or practical impact on the trial and the trial participants. In this respect, we take post-treatment access to successful interventions for the relevant populations for granted, although this may be a very challenging issue in specific cases.

Essential literature on research involving human subjects is listed in Table 2. 
Kuepfer \& Burri, Reflections on clinical research in sub-Saharan Africa

Table 2. Essential literature on research including human subjects.

Documents

International Conference on Harmonization / ICH Harmonized tripartite guideline: Guideline for Good Clinical Practice GCP E6 (3)

World Medical Association Declaration of Helsinki (http://www.wma.net/e/policy/b3.htm)

Council for International Organizations of Medical Sciences / CIOMS: International ethical guidelines for biomedical research involving human subjects (in collaboration with WHO) (21)

Nuffield Council on Bioethics: The ethics of research related to healthcare in developing countries (22)

National Bioethics Advisory Commission / NBAC: Ethical and Policy Issues in International Research: Clinical Trials in Developing Countries (23)

\section{Ethics committees}

The ethical clearance by an independent ethics committee in the host as well as in the sponsoring country is one of the key requirements. The first African ethics committee was established in South Africa, 1967 (24). But in 2005, 36\% of the WHO African Region countries still had no ethics committees. Capacity development in the area of research ethics was promoted (25) and the establishment of ethics committees and the provision of training and capacity building is today actively supported by, for example, the PanAfrican Bioethics Initiative (PABIN) (http://www.pabin.org) as a member of the global Strategic Initiative for Developing Capacity in Ethical Review (SIDCER) and the South African Research Ethics Training Initiative (SARETI) funded by the Fogarty International Center of the US NIH (http://www.fic.nih.gov). However, many committees are not yet fully functional due to lack of funding, infrastructure, training, standard operating procedures and sometimes simply the lack of political commitment from the governments.

Besides national ethical clearance of the research project in the host country we strongly encourage ethical clearance by local ethics committees. Local ethics committees can best represent the cultural, political and economic values of the region and can act as a hinge between community needs and research priorities. The value of the implementation of a local ethics committee depends on the number of clinical research projects conducted in the particular area/region and will likely be linked to a well-established research center.

Today, the lack of capacity of National Regulatory Authorities (NRA) leads to the situation that ethics committees often fill the role of local drug regulators, too. However, not only for this reason, strengthening of NRA capacities has a very high priority. Efforts must include the authorization and monitoring of clinical trials, the evaluation of clinical data for product registration, and quality control and pharmacovigilance of medicinal products.

In view of this formidable task, systems supporting NRAs and optimizing the use of regional resources and expertise are needed. An example is the WHO African Vaccine Regulatory Forum (AVAREF) which can be considered as an "ad-hoc" scientific advisory body that can help regulators to make an informed regulatory decision with regards to authorizations of clinical trials, evaluation of registration dossiers or any other challenging issues regarding evaluation of vaccines (11).

\section{Informed consent and assent}

Patient information and written informed consent are two of the most sensitive and complex parts of clinical research. This is true for all clinical research conducted but even more so in sub-Saharan Africa. In contrast to Western culture, communal consciousness and living is the norm in many African societies and for decision-making processes, the importance of community leaders and families cannot be eroded (26). Patient information forms which are long, complex and sometimes inappropriate in the cultural context where they are used, may confuse, rather than inform, participants and can be refused by the local Ethics committees (27). Balancing completeness versus simplicity is a real challenge in the preparation of patient information. An appropriate patient information and informed consent procedure is key in the 
prevention of the therapeutic misconception; when participants believe that they are receiving a new form of treatment rather than participating in research (28).

If children are involved, the parents or the legal guardian must give consent. However, children capable of making decisions (depending on the society and the disease of an age of 7 or older) should be asked for their assent which must be respected if the response is negative (29).

A further sensitive issue is the requirement for written informed consent (3). In certain cultures or situations, signing of a consent form has very negative connotations and may even be rejected (22). Whether written consent forms are adequate for use with illiterate patients has also been debated and the National Bioethics Advisory Commission has issued a recommendation to waive written consent in such situations (23) and to replace it with an alternative appropriate process which must be specified in the protocol (e.g. independent witnesses).

Finally, if the language in which the information is to be provided is different from the one used for the protocol, the text must be translated to a locally spoken language and should be back-translated to detect and rule out misunderstandings. This process may be particularly difficult and tedious in settings where indigenous languages with a limited modern word pool are spoken and where the translation and back translation process requires particular attention. If a language is not written, the information must be read and explained from a sheet written in an alternative official language. In this case, the presence of a knowledgeable witness seems to be appropriate.

\section{Indemnities and undue inducement}

Particular attention is required for the adequate indemnification of study participants. One has to be aware of the coercion or undue inducement (30) of overpaid indemnities and of exclusive payments linked to completion of the trial. It is clear that the patients in resource-poor settings need to receive a fair compensation for their efforts such as transportation or sometimes for the working time lost during hospitalization. The local representatives often opt for paying in kind, particularly in rural settings. However, there is a fine line and one should be aware that trial or project sites can comply with the research proposals because they bring work, salaries, technology transfer and materials to their locations.

\section{Publication of results and trial registration}

The publication of positive and negative trial results is an ethical requirement as stated in the Declaration of Helsinki (http://www.wma.net/e/policy/b3.htm). However, in reality the publication of results is frequently delayed or even omitted. Corporate involvement in clinical research may lead to the exclusive disclosure of results to regulators. The very long follow-up periods typical to a number of neglected diseases such as human African trypanosomiasis, contribute to extensive delays in the publication of data. The increasing acceptance of the importance of public information and the reduction of selective reporting is reflected in the mandatory registration of clinical trials. In 2004, the International Committee of Medical Journal Editors published a respective joint editorial: before first patient enrolment any clinical trial must be registered in a public database to allow publication. There are various public registries for clinical trials but since 2005 the "WHO International Clinical Trial Registry Platform" (http://www.who.int/trialsearch/) has taken the lead. As another mechanism which might assist the timely publication of data on diseases requiring a long patient follow-up, we suggest discussion of the option to separately publish the safety and efficacy data in two linked publications. Table 3 summarizes the minimum ethical standards to be met at local, national and sponsor level. 
Table 3. Minimum ethical standards for research involving human subjects.

\begin{tabular}{|c|c|c|}
\hline LOCAL LEVEL & NATIONAL LEVEL & SPONSOR LEVEL \\
\hline $\begin{array}{l}\text { Staff understanding of the } \\
\text { importance of a correct patient } \\
\text { information and a correct } \\
\text { informed consent process } \\
\text { Respect of right for anonymity } \\
\text { of trial participants } \\
\text { Community information and } \\
\text { involvement } \\
\text { Fair information of all potential } \\
\text { participants (complete, simple } \\
\text { wording) } \\
\text { Consent at relevant levels } \\
\text { Individual consent of all } \\
\text { participant } \\
\text { Written consent as a standard } \\
\text { option; alternatives with } \\
\text { sufficient justification where } \\
\text { necessary } \\
\text { Fair compensation for trial } \\
\text { participation }\end{array}$ & $\begin{array}{l}\text { Timely ethical review by } \\
\text { designated national or thematic } \\
\text { Ethics committee } \\
\text { Timely approbation of cleared } \\
\text { trial by drug authorities or } \\
\text { Ministry of Health and } \\
\text { controlled importation of the } \\
\text { investigational product }\end{array}$ & $\begin{array}{l}\text { Study design respecting the } \\
\text { local situation, capacities and } \\
\text { cultural constraints } \\
\text { Ethical review in sponsor (or } \\
\text { CRO) home-country unless host } \\
\text { country IEC is certified } \\
\text { Trial registration }\end{array}$ \\
\hline
\end{tabular}

\section{Implementation and conduct of trials in resource-limited environments}

In the case that a clinical research project is carried out in collaboration with a rural trial site, the sponsor is confronted with challenges that do not exist in other settings. The absence of well educated health personnel, high staff turnover, poor infrastructure, lack of standard operating procedures and difficult accessibility are common. Often the situation is complicated by an unstable security situation created by political conflicts. Importantly, traditional beliefs and stigma have a strong impact on the behavior of the population and health staff, and may influence the research activities.

For the conduct of clinical trials in such situations, centre assessment and site selection are of paramount importance. The capacities and infrastructures very often must be built up and maintained on a continuous basis. Issues which are practically unknown in the implementation of trials in Western settings must be considered: the presence of an educated physician and other staff members, appropriate laboratories including (stable) energy supply, laboratory staff training and the installation of communication tools may be issues. The appropriate level of site improvements should be balanced between the requirements of the trial and the maximum capacities of the staff.

Quite often the questions asked in a protocol have to be adapted to the limited possibilities of the sites. It is wiser to focus on the key topics and to refrain from obtaining information on all details. The basic principle of GCP, that anything not documented does not exist, necessitates a thorough and clean recording of data and the creation of an appropriate and easy-to-monitor filing and recording system. Such consistent systems rarely exist in rural health facilities where records are usually kept in various books and booklets. Furthermore, long-term storage of the study and patient records must be assured, which can be problematic considering heat, humidity and the often precarious room situations.

Dedicated pharmacies and adequate clean and air-conditioned storage with limited access are often nonexistent in such facilities and practical solutions must be found to protect the experimental product from misuse, to guarantee its quality and to account for its receipt, use and return or destruction.

A difficult and critical issue is the reporting of adverse and serious adverse events (SAEs). If not stressed, adverse events may not be recorded at all as they are not considered unusual. For SAEs it is important to (i) convey the difference between severeness (clinical term) and seriousness (technical and legal term) and (ii) to implement a reliable, monitorable communication system that allows timely reporting of SAEs to the sponsor/contract research organization and the Ethics committee. 
Clinical data management (CDM) is a particularly delicate topic. Under the new regulations CDM has become a highly regulated field. Specialized software and tedious validation of the programs, systems, data transfer and trial setup are necessary. To avoid post-trial or even approval difficulties the CDM for multi-center, multi-country trials is usually concentrated at dedicated contract research companies. But centralized data collection and storage may conflict with the intentions and ambitions of the local investigators. In particular, sites with links to a demographic surveillance system or which routinely run large-scale epidemiological studies may have sophisticated equipment and statistical knowledge and opt for local CDM. In our experience with such situations, a pragmatic approach is advantageous, since inconsistent data quality and complicated trial structures cannot be in anyone's interest.

Final study reports to the regulatory authorities should be written by professional scientific writers but the sponsors should clearly recognize the needs and interests of their partners to publish results and their active involvement should already be defined in the study protocol.

For the control of certain diseases, e.g. human African trypanosomiasis and onchocerciasis, specialized mobile teams visiting the population at risk are necessary. Those teams have to be integrated in the work flow of the respective clinical trials and can be used for the information of the population, for prescreening of potential patients and even enroll participants in a trial. To maintain a coherent technical standard, a strong presence of technical advisors, supervisors and specialized monitors familiar with the disease, the local situation and the language, and intensive external support in site management have to be warranted. Typically, this approach is only possible through a joint venture of the implementing organization with the responsible national and/or district authority. Such partnerships have to be built and preserved carefully, as the enrolment in trials on neglected diseases tends to be very slow. An example for this is given in the accounts of the Phase II trial for Moxidectin against onchocerciasis (31).

In summary, the conduct of clinical trials in rural settings is certainly not a low-cost option and entails particular challenges. Table 4 summarizes the minimum standards required to conduct GCP-compliant trials.

Table 4. Minimum standards for the conduct of clinical trials according to the International Conference on Harmonization/ Good Clinical Practice (3).

\begin{tabular}{|c|c|c|}
\hline LOCAL LEVEL & NATIONAL LEVEL & SPONSOR LEVEL \\
\hline $\begin{array}{l}\text { The presence of educated medical } \\
\text { staff and nursing care } \\
\text { Capacities for documentation and } \\
\text { archiving } \\
\text { Assurance of confidentiality } \\
\text { Accurate reporting and verification } \\
\text { of the data } \\
\text { Adherence to the protocol } \\
\text { Version control for protocol and } \\
\text { informed consent } \\
\text { Correct, timely reporting and } \\
\text { handling of adverse and serious } \\
\text { adverse drug reactions } \\
\text { Infrastructures for correct storage } \\
\text { and use of the investigational } \\
\text { medicinal product }\end{array}$ & $\begin{array}{l}\text { Monitoring and surveillance of } \\
\text { ongoing projects }\end{array}$ & $\begin{array}{l}\text { Responsibility for quality } \\
\text { assurance and quality control } \\
\text { Qualified personnel for the trial } \\
\text { design, trial management and } \\
\text { medical expertise } \\
\text { Trial registration } \\
\text { Insurance for participants } \\
\text { Financing } \\
\text { Notification and submission to } \\
\text { regulatory authorities } \\
\text { Information on investigational } \\
\text { product (investigators brochure) } \\
\text { Manufacturing, packaging, } \\
\text { labeling, (coding) and supply of } \\
\text { investigational product } \\
\text { Notification of end of study } \\
\text { Renorting }\end{array}$ \\
\hline
\end{tabular}




\section{Access and delivery}

This topic, although of paramount importance for every intervention, goes beyond the scope of this paper and we will only summarily examine it.

\section{Post marketing studies and pharmacovigilance}

When a marketing application is filed for regulatory approval, the documentation for a new drug will typically comprise a few thousand patients with data mainly collected through Phase I to III trials (32). Although these data are considered to be sufficient to describe the safety and efficacy profile of a new compound, at this stage there is no data about the effectiveness of the drug in the real-life situation. Questions about unforeseen drug interaction, rare adverse events, effects of poor patient compliance and dynamics of resistances have not yet been addressed. This information is needed for the safe use of a drug, particularly in high risk groups such as pregnant and lactating women, children, malnourished and HIV-infected patients. In addition, the information is an important element in making a decision whether a new intervention should be added to the essential drug list of a country. At the global level, the WHO program for international drug monitoring at the Uppsala monitoring centre collates adverse drug reaction reports via the national pharmacovigilance centers of the 81 member countries (www.who-umc.org). Currently there are only six sub-Saharan African countries (South Africa, Zimbabwe, Tanzania, Mozambique, Nigeria and Ghana) that are full members of the program. In fact, less than $27 \%$ of lower middle income countries have national pharmacovigilance systems registered with the WHO program, compared with $96 \%$ of the high income countries in the Organization for Economic Co-operation and Development. The main reasons for this are lack of resources, infrastructure and expertise. Thus, although access to medicines is increasing in developing countries, there is a danger that their risk-benefit profiles in indigenous populations will not be fully monitored (33). To make up for this shortcoming of local data, at least substantial Phase IIIb or drug utilization trials should be conducted.

\section{Access}

The effectiveness of an intervention depends on many factors which may be linked to a product or be parameters of the health system, such as affordable market price, distribution channels and their accessibility, sustained availability of the product, quality control, information for health care professionals and the general public, and correct diagnosis. At each level, those involved may have conflicting interests, and poor populations are the first to suffer the effects of frail links in this long chain (34).

Recent experience indicates that the drugs developed in PDPs have a faster introduction to the market. This puts a lot of pressure on public health systems and currently, many projects fail because the health systems are not strong enough to deliver new interventions to the target populations. Also the Global Fund to fight AIDS, tuberculosis and malaria (http://www.theglobalfund.org) recognizes this need and supports the strengthening of health systems (35). An important aspect is health information. It is recognized that inadequate access to information is a significant factor in development, and particularly in health care development. Despite major global progress in access to information during the last decade, there is little evidence that health professionals, especially those working in rural primary health care, are better informed than they were 10 years ago (36). The unequal distribution of health care between developed and developing countries is matched by a similar unequal distribution of health information (37) and the 10/90 gap in health research probably translates into a 1/99 gap in health information (36).

\section{The way forward}

The development and provision of new interventions to prevent, diagnose, treat and control diseases that affect the poorest and most vulnerable populations finally is receiving global attention and support. Today, numerous initiatives, consortia and PDPs are engaged in those activities and the funding has reached unprecedented dimensions. This results in a large number of ongoing research projects and the proportion of clinical trials being truly performed according to GCP is increasing. This development has already led to tangible results. For instance, several new chemical entities for the treatment of malaria have been or will be registered shortly by first tier drug authorities. A number of new research centers in sub-Saharan Africa have emerged and are gaining momentum. A strong generation of scientists is 
developing and is reasonably well-supported through various competitive fellowship grants. This also opens the possibility that researchers will increasingly see career opportunities at home which may reduce the "brain drain" effect and with this, innovation and leadership will be strengthened in the long term. So, can we lean back and wait for better days dawning? There are still serious gaps and short-comings which will require enormous effort to be overcome. It now seems critical to maintain impetus in the research area and to continue the processes started at all levels. This will not be achieved solely by continuing funding but also through genuine political interest and backing by the governments and institutional leaders of the developing countries. From the R\&D point of view, there is a need for the technical strengthening of all divisions or drug regulatory authorities, participatory involvement of research scientists at institutional levels and the reduction of artificial logistical hurdles.

The treatment of many of the most neglected diseases is still dreadful and this will certainly still need increasing attention. However, the transition of health (i.e. the epidemiologic shift from infectious to chronic diseases particularly in urban areas) will incontestably create new challenges, but potentially also new opportunities for the research centers involved in R\&D.

\section{Acknowledgements}

We would like to thank Marcel Tanner and Juerg Utzinger for discussions and valuable input. We are grateful to Benjamin Dahl for critical reading and correction of the manuscript. 


\section{References}

1. MSF/DNDi. Fatal Imbalance: The crisis in Research and Development for Drugs for Neglected Diseases. Geneva: Médecins Sans Frontières Access to Essential Medicines Campaign, Drugs for Neglected Diseases Working Group; 2001.

2. Moran M. A breakthrough in R\&D for neglected diseases: new ways to get the drugs we need. PLoS Med. 2005 Sep;2(9):e302.

3. ICH/GCP. ICH harmonized tripartite guideline, Guideline for good clinical practice E6 (R1). Surrey: Canary Publications, UK; 1996.

4. Hotez P, Stoever K, Fenwick A, Molyneux D, Savioli L. The Lancet's chronic diseases series. Lancet. 2006 Feb 18;367(9510):563-4; author reply 4-5.

5. Perrin D, Scheer A, Wells T. Collaborating to find new approaches to tropical diseases. European Pharmaceutical Review. 2006 23.05.2006;3:1-4.

6. Nwaka S, Ridley RG. Virtual drug discovery and development for neglected diseases through public-private partnerships. Nat Rev Drug Discov. 2003 Nov;2(11):919-28.

7. Trouiller P, Olliaro P, Torreele E, Orbinski J, Laing R, Ford N. Drug development for neglected diseases: a deficient market and a public-health policy failure. Lancet. 2002 Jun 22;359(9324):2188-94.

8. Gelb MH, Hol WG. Parasitology. Drugs to combat tropical protozoan parasites. Science. 2002 Jul 19;297(5580):343-4.

9. Noedl H, Se Y, Schaecher K, Smith BL, Socheat D, Fukuda MM. Evidence of artemisinin-resistant malaria in western Cambodia. N Engl J Med. 2008 Dec 11;359(24):2619-20.

10. Chirac P, Torreele E. Global framework on essential health R\&D. Lancet. 2006 May 13;367(9522):1560-1.

11. WHO. Human African trypanosomiasis (sleeping sickness): epidemiological update. Weekly epidemiological record. 2006 24. Februar(TRS881):69-80.

12. Kesselheim AS. Drug development for neglected diseases - the trouble with FDA review vouchers. N Engl J Med. 2008 Nov 6;359(19):1981-3.

13. EMEA. Guidance for companies requesting scientific advice or protocol assistance. Guideline. London: European Medicine Agency; 2005 17. November.

14. Garner C. Dealmaking and Intellectual Property Management for Public Interest. Meeting Report. Bethesday, Mayland: The Initiative on Public-Private Partnerships for Health (IPPPH), The Centre for Management of Intellectual Property in Health Research and Development (MIHR), Aeras Global TB Vaccine Foundation; 2004 29-30. Nov.

15. Hertzfeld H, Link A, Vonortas N. Intellectual property protection mechanisms in research parnerships. Research Policy. 2006;35:825-38.

16. Whitworth JA, Kokwaro G, Kinyanjui S, Snewin VA, Tanner M, Walport M, et al. Strengthening capacity for health research in Africa. Lancet. 2008 Nov 1;372(9649):1590-3.

17. Gyapong J, Ofori-Adjei D. Capacity building for relevant health research in developing countries. Knowledge on the Move; 2008 26.02.2008; The Hague: Netherlands organization for international cooperation in higher education (nuffic); 2008.

18. Angell M. The ethics of clinical research in the Third World. N Engl J Med. 1997 Sep 18;337(12):847-9.

19. FDA. Human subject protection; foreign clinical studies not conducted under an Investigational New Drug Application. CDER 200482 ed: Food and Drug Administration; 2008. p. 22800-16.

20. White NJ. Editorial: clinical trials in tropical diseases: a politically incorrect view. Trop Med Int Health. 2006 Oct;11(10):1483-4.

21. CIOMS. International ethical guidelines for biomedical research involving human subjects. Bull Med Ethics. $2002 \operatorname{Oct}(182): 17-23$.

22. NCOB. The ethics of research related to healthcare in developing countries. Report. London: Nuffield Council on Bioethics; 2002 April 2002.

23. NBAC. Ethical and Policy Issues in International Research: Clinical Trials in Developing Countries. Bethesda, Maryland: National Bioethics Advisory Commission; 200130.04.

24. Kass NE, Hyder AA, Ajuwon A, Appiah-Poku J, Barsdorf N, Elsayed DE, et al. The structure and function of research ethics committees in Africa: a case study. PLoS Med. 2007 Jan;4(1):e3.

25. Kirigia JM, Wambebe C, Baba-Moussa A. Status of national research bioethics committees in the WHO African region. BMC Med Ethics. 2005 Oct 20;6:E10. 
26. Bhutta ZA. Beyond informed consent. Bull World Health Organ. 2004 Oct;82(10):771-7.

27. Mueller J, Schellenberg D, Owens S. Third-World Barriers - Getting past consent and ethical issues endemic in underserved populations to ensure quality GCP. Applied Clinical Trials. 2007 01.10.2007; October 2007:58-64.

28. Fairlamb AH, Henderson GB, Cerami A. Trypanothion is the primary target for arsenical drugs against african trypanosomes. Proceedings of the National Academy of Sciences of the United States of America. 1989;86:2607-11.

29. Broome ME. Consent (assent) for research with pediatric patients. Semin Oncol Nurs. 1999 May;15(2):96-103.

30. Emanuel EJ, Currie XE, Herman A. Undue inducement in clinical research in developing countries: is it a worry? Lancet. 2005 Jul 23-29;366(9482):336-40.

31. Kuesel A, Lazdins J. A new drug for river blindness? TDRnews. 2007 December 2007;79:14-9.

32. Rawlins MD. Pharmacovigilance: paradise lost, regained or postponed? The William Withering Lecture 1994. J R Coll Physicians Lond. 1995 Jan-Feb;29(1):41-9.

33. Pirmohamed M, Atuah KN, Dodoo AN, Winstanley P. Pharmacovigilance in developing countries. Bmj. 2007 Sep 8;335(7618):462.

34. Pecoul B, Chirac P, Trouiller P, Pinel J. Access to essential drugs in poor countries: a lost battle? Jama. 1999 Jan 27;281(4):361-7.

35. WHO/GFATM. The Global Fund Strategic Approach to Health Systems Strengthening. Report. Geneva: The Global Fund to fight AIDS, Tuberculosis and Malaria; 2007 September. Report No.: WHO/HSS/2007.1.

36. Godlee F, Pakenham-Walsh N, Ncayiyana D, Cohen B, Packer A. Can we achieve health information for all by 2015? Lancet. 2004 Jul 17-23;364(9430):295-300.

37. Katikireddi SV. HINARI: bridging the global information divide. Bmj. 2004 May 15;328(7449):1190-3. 